\title{
Research on Aircraft Attack Angle Control Considering Servo-Loop Dynamics
}

\author{
Xiaodong Liu, ${ }^{1,2}$ Yan Wang, ${ }^{3}$ Wanwei Huang, ${ }^{1,2}$ and Zongxia Jiao ${ }^{3}$ \\ ${ }^{1}$ Beijing Aerospace Automatic Control Institute, Beijing 100854, China \\ ${ }^{2}$ Science and Technology on Aerospace Intelligent Control Laboratory, Beijing 100854, China \\ ${ }^{3}$ School of Automation Science and Electrical Engineering, Beihang University, Beijing 100191, China \\ Correspondence should be addressed to Xiaodong Liu; lxd@asee.buaa.edu.cn
}

Received 27 September 2014; Revised 31 December 2014; Accepted 31 December 2014

Academic Editor: Ronald M. Barrett

Copyright (C) 2015 Xiaodong Liu et al. This is an open access article distributed under the Creative Commons Attribution License, which permits unrestricted use, distribution, and reproduction in any medium, provided the original work is properly cited.

\begin{abstract}
This paper presents a novel robust attack angle control approach, which can effectively suppress the impacts from system uncertainties and servo-loop dynamics. A second-order linear model of electromechanical servo loop is considered in the modeling and design processes. With regard to the block-structure models facing attack angle control, the multiple robust surfaces and dynamic surface control (DSC) approaches are both employed. By means of Lyapunov function method, the stability conditions of attack angle control systems are, respectively, given without/with considering the servo-loop dynamics in design process. Computer simulation results present that, compared with the attack angle control scheme which does not consider the servo-loop dynamics in design process, the proposed scheme can guarantee that the whole attack angle control system possesses the better comprehensive performances. Moreover, it is easy to be realized in engineering application.
\end{abstract}

\section{Introduction}

In aircraft attitude control system, the existing system uncertainties, including modeling errors, parameter perturbations, and external disturbances, can badly affect the flight performances and even lead to the failure of flight task [1]. As a result, the impacts of system uncertainties on control performances must be highly valued and then effectively suppressed in the design process of aircraft attitude control law [2-4]. Besides that, the servo-loop dynamics, which is not absolutely ideal, can also affect the performances of the whole attitude control system. Concretely speaking, if the attitude control law is previously designed without considering the servo-loop dynamics, the whole attitude control system will not keep the satisfactory performance when the servo loop is added into the closed-loop system. Therefore, it is necessary to explore the robust attitude control methods with consideration of servo-loop dynamics, in order to further improve the comprehensive performance of the whole attitude control system. Taking the pitching channel into consideration, the attack angle control approach with consideration of a second-order linear model of electromechanical servo loop will be explored in this paper.

With regard to the aircraft flight system, the multiple robust surfaces method combined with backstepping method is generally employed $[1,4-6]$. However, the traditional integrator backstepping technique suffered from the problem of "explosion of terms," which can lead to the performance degradation (even the serious instability) of flight control system. In order to solve this problem, Swaroop et al. [7] proposed a kind of dynamic surface control (DSC) method, where the model is not directly differentiated through introducing some groups of low-pass filters. As a result, DSC approach is more appropriately used in the control system with multilevel framework than traditional backstepping approach, and it has been widely applied in the area of flight guidance and control [8-10]. In this paper, taking the pitching channel as an example, DSC method will be employed into the design of attack angle control law on the condition that the servo-loop dynamics is considered. In addition, because 
sliding mode control (SMC) possesses a series of attractive characteristics, especially the strong robust property against parameter perturbations and external disturbances [11-13], it has been paid more attentions and widely applied in aircraft control areas $[3,6]$. Based on this, the SMC theory will be also introduced in the design process of subsystem control.

The remainder of this paper is organized as follows. Section 2 provides the design models facing attack angle control without/with considering the servo-loop dynamics. Based on as-built models, the dynamic surface attack angle control laws are, respectively, designed without/with considering the servo-loop dynamics in Section 3. In Section 4, the computer simulation results are presented; meanwhile the conclusions and future works are given in Section 5.

\section{Problem Description}

Only considering the pitching channel of aircraft, the attitude motion equations are shown as follows [1]:

$$
\begin{aligned}
\dot{\alpha} & =-\frac{C_{Y} q S}{m V}+\omega_{z 1}+\frac{g \cos \theta}{V}, \\
\dot{\omega}_{z 1} & =\frac{C_{z 1} q S L}{J_{z 1}},
\end{aligned}
$$

where $\alpha$ and $\theta$, respectively, denote the attack angle and the flight path angle; $m$ and $V$, respectively, denote the mass and the velocity of aircraft; $g$ denotes the acceleration of gravity; $\omega_{z 1}$ and $J_{z 1}$, respectively, denote the pitching angular speed and pitching moment of inertia; $q=0.5 \rho V^{2}$ denotes the dynamic pressure and $\rho$ denotes the air density; $S$ and $L$, respectively, denote the reference area and the reference length for calculating the aerodynamic forces and aerodynamic moments. In addition, $C_{Y}$ denotes the aerodynamic lift coefficient, $C_{z 1}$ denotes the pitching moment coefficient, and the expressions of them in a certain flight extent are shown as follows:

$$
\begin{gathered}
C_{Y}=C_{Y}^{\alpha} \alpha+C_{Y}^{\delta_{z}} \delta_{z}, \\
C_{z 1}=C_{z 1}^{\alpha} \alpha+C_{z 1}^{\delta_{z}} \delta_{z},
\end{gathered}
$$

where $\delta_{z}$ denotes the output angle of servo loop, $C_{Y}^{\alpha}$ denotes the aerodynamic lift coefficient with respect to $\alpha$, and $C_{Y}^{\delta_{z}}$ denotes the aerodynamic lift coefficient with respect to $\delta_{z}$. Meanwhile, $C_{z 1}^{\alpha}$ denotes the pitching moment coefficient with respect to $\alpha$, and $C_{z 1}^{\delta_{z}}$ denotes the pitching moment coefficient with respect to $\delta_{z}$. Note that (1)-(2) can be used to construct the actual model in simulation.

Subsequently, when the servo-loop dynamics is not considered in the design period, the design model facing attack angle control is obtained as follows:

$$
\begin{aligned}
\dot{\alpha} & =a_{1} \alpha+\omega_{z 1}+f_{\theta}+d_{\alpha}, \\
\dot{\omega}_{z 1} & =a_{2} \alpha+b_{2} \delta_{z}+d_{\omega},
\end{aligned}
$$

where $d_{\alpha}$ and $d_{\omega}$ denote system uncertainties, which involves the modeling errors from the actual model to the design model. Moreover,

$$
\begin{aligned}
& a_{1}=-\frac{C_{Y}^{\alpha} q S}{m V}, \\
& f_{\theta}=\frac{g \cos \theta}{V}, \\
& a_{2}=\frac{C_{z 1}^{\alpha} q S L}{J_{z_{1}}}, \\
& b_{2}=\frac{C_{z 1}^{\delta_{z}} q S L}{J_{z_{1}}} \neq 0 .
\end{aligned}
$$

For DC electromechanical servo system, it is usually simplified into a second-order linear model, which is shown as follows:

$$
G(s)=\frac{\delta_{z}(s)}{\delta_{z d}(s)}=\frac{\omega_{n}{ }^{2}}{s^{2}+2 \xi_{n} \omega_{n} s+\omega_{n}{ }^{2}},
$$

where $\omega_{n}$ and $\xi_{n}$, respectively, denote the angular frequency of natural oscillation and the damping coefficient of servo loop and $\delta_{z d}$ denotes the command input of servo loop. Moreover, the value selection of $\omega_{n}$ and $\xi_{n}$ should reflect the demands on the bandwidth and the dynamic properties of servo loop.

In fact, (5) can be rewritten in the time-domain form, which is shown as follows:

$$
\ddot{\delta}_{z}=-2 \xi_{n} \omega_{n} \dot{\delta}_{z}-\omega_{n}^{2} \delta_{z}+\omega_{n}^{2} \delta_{z d}+d_{\delta}
$$

where $d_{\delta}$ denotes the system uncertainty.

Therefore, when the servo-loop dynamics is considered in the design period, the design model facing attack angle control will be transformed into

$$
\begin{aligned}
\dot{\alpha} & =a_{1} \alpha+\omega_{z 1}+f_{\theta}+d_{\alpha}, \\
\dot{\omega}_{z 1} & =a_{2} \alpha+b_{2} \delta_{z}+d_{\omega}, \\
\ddot{\delta}_{z} & =-2 \xi_{n} \omega_{n} \dot{\delta}_{z}-\omega_{n}{ }^{2} \delta_{z}+\omega_{n}{ }^{2} \delta_{z d}+d_{\delta} .
\end{aligned}
$$

At this time, we can see that the original design model (3) with two-layer structure changes into another one (7) with threelayer structure, which directly reflects the deference between the modeling processes without/with considering the servoloop dynamics.

The establishment of design models (3) and (7) lays the foundation for the next design of aircraft attack angle control laws.

\section{Dynamic Surface Attack Angle Controller Design}

With regard to a class of system with strict feedback form, just as system (3) and system (7), multiple robust surfaces method combined with backstepping method is generally employed. 
However, the traditional integrator backstepping technique suffered from the problem of "explosion of terms"; therefore the other improved control method-DSC-will be applied in this section, where the model is not directly differentiated through introducing some groups of low-pass filters.

Owing to the simple design and strong robust property of SMC, it will be introduced in the design process of subsystem control. Furthermore, the reaching law approach [14, 15] can effectively guarantee the better dynamic performance of reaching process; therefore it will be employed to construct the SMC scheme in this section.

The following two assumptions are useful in the next design and analysis.

Assumption 1. The uncertainty terms in system are all bounded; that is, $\left|d_{\alpha}\right| \leq \Delta_{\alpha},\left|d_{\omega}\right| \leq \Delta_{\omega}$, and $\left|d_{\delta}\right| \leq \Delta_{\delta}$.

Assumption 2. When the DSC method is applied, the filter output $y_{f_{o}}$ is in a neighborhood of the filter input $y_{f i}$; that is, $\left|y_{f o}-y_{f i}\right| \leq \Delta_{f}$.

3.1. Without Consideration of Servo-Loop Dynamics. Aiming at the two-layer design model (3), the dynamic surface attack angle control law can be designed as in the following steps.

Step A1. Design the robust surface variable for the 1st-layer subsystem:

$$
s_{1}=\alpha-\alpha_{d}
$$

where $\alpha_{d}$ denotes the command of attack angle.

Step A2. Calculate the virtual command of the 2nd-layer subsystem:

$$
\begin{array}{r}
\omega_{z 1 d}=-a_{1} \alpha+\dot{\alpha}_{d}-k_{1} s_{1}-q_{1} \operatorname{sgn}\left(s_{1}\right)-f_{\theta}, \\
k_{1}>0, \quad q_{1}>0 .
\end{array}
$$

Step A3. In order to get the differential information of $\omega_{z 1 d}$, we introduce a filter:

$$
\eta \dot{\omega}_{z 1 c}+\omega_{z 1 c}=\omega_{z 1 d}, \quad \omega_{z 1 c}(0)=\omega_{z 1 d}(0), \quad \eta>0 .
$$

Based on Assumption 2, we can get $\omega_{z 1 c}=\omega_{z 1 d}+d_{f \omega}$, where $\left|d_{f \omega}\right| \leq \Delta_{f \omega}$. In fact, we can adjust the parameter $\eta$ to make the boundary $\Delta_{f \omega}$ arbitrarily small.

Step A4. Design the robust surface variable for the 2nd-layer subsystem:

$$
s_{2}=\omega_{z 1}-\omega_{z 1 c} .
$$

Step A5. Calculate the command input of servo loop:

$$
\begin{array}{r}
\delta_{z d}=\frac{-a_{2} \alpha-k_{2} s_{2}-q_{2} \operatorname{sgn}\left(s_{2}\right)+\dot{\omega}_{z 1 c}}{b_{2}}, \\
k_{2}>0, \quad q_{2}>0 .
\end{array}
$$

Theorem 3. When the servo-loop dynamics is not considered, by means of Steps A1-A5, the actual attack angle $\alpha$ will tend to the desired one $\alpha_{d}$, if the following conditions in (13) are all satisfied:

$$
\begin{aligned}
k_{1} k_{2} & >\frac{1}{4}, \\
q_{1} & >\Delta_{\alpha}+\Delta_{f \omega}, \\
q_{2} & >\Delta_{\omega} .
\end{aligned}
$$

Proof. Choose a Lyapunov function as follows:

$$
V=\frac{1}{2} s_{1}^{2}+\frac{1}{2} s_{2}^{2}
$$

Then, with the differential operation on (14), one can get

$$
\dot{V}=\dot{s}_{1} s_{1}+\dot{s}_{2} s_{2}
$$

According to (3), (8), and (11), one can get

$$
\begin{aligned}
\dot{s}_{1} & =\dot{\alpha}-\dot{\alpha}_{d}=a_{1} \alpha+\omega_{z 1}+f_{\theta}+d_{\alpha}-\dot{\alpha}_{d} \\
& =a_{1} \alpha+s_{2}+\omega_{z 1 c}+f_{\theta}+d_{\alpha}-\dot{\alpha}_{d} .
\end{aligned}
$$

Owing to $\omega_{z 1 c}=\omega_{z 1 d}+d_{f \omega}$, (16) can be transformed into

$$
\dot{s}_{1}=a_{1} \alpha+s_{2}+\omega_{z 1 d}+f_{\theta}+d_{s 1}-\dot{\alpha}_{d}
$$

where $d_{s 1}=d_{\alpha}+d_{f \omega}$ is bounded with $\left|d_{s 1}\right| \leq \Delta_{s 1}=\Delta_{\alpha}+\Delta_{f \omega}$.

Substituting (9) into (17), one can get

$$
\dot{s}_{1}=s_{2}-k_{1} s_{1}-q_{1} \operatorname{sgn}\left(s_{1}\right)+d_{s 1} .
$$

In similar, according to (3), (11), and (12), one can get

$$
\begin{aligned}
\dot{s}_{2} & =\dot{\omega}_{z 1}-\dot{\omega}_{z 1 c}=a_{2} \alpha+b_{2} \delta_{z d}+d_{\omega}-\dot{\omega}_{z 1 c} \\
& =-k_{2} s_{2}-q_{2} \operatorname{sgn}\left(s_{2}\right)+d_{\omega} .
\end{aligned}
$$

Substituting (18)-(19) into (15), we can get

$$
\begin{aligned}
\dot{V}= & {\left[s_{2}-k_{1} s_{1}-q_{1} \operatorname{sgn}\left(s_{1}\right)+d_{s 1}\right] s_{1} } \\
& +\left[-k_{2} s_{2}-q_{2} \operatorname{sgn}\left(s_{2}\right)+d_{\omega}\right] s_{2} \\
= & -k_{1} s_{1}{ }^{2}-k_{2} s_{2}{ }^{2}+s_{1} s_{2}-q_{1}\left|s_{1}\right|+d_{s 1} s_{1}-q_{2}\left|s_{2}\right| \\
& +d_{\omega} s_{2} \\
\leq & -2 \sqrt{k_{1} k_{2}}\left|s_{1} s_{2}\right|+\left|s_{1} s_{2}\right|-q_{1}\left|s_{1}\right|+\Delta_{s 1}\left|s_{1}\right| \\
& -q_{2}\left|s_{2}\right|+\Delta_{\omega}\left|s_{2}\right| \\
= & -\left(2 \sqrt{k_{1} k_{2}}-1\right)\left|s_{1} s_{2}\right|-\left(q_{1}-\Delta_{s 1}\right)\left|s_{1}\right| \\
& -\left(q_{2}-\Delta_{\omega}\right)\left|s_{2}\right| .
\end{aligned}
$$


Then, under the satisfaction of conditions in (13), we can further get that $\dot{V} \leq 0$ always exists. Moreover, when and only when both $s_{1}=0$ and $s_{2}=0$ coexist, $\dot{V}=0$ is tenable. As a result, $s_{1} \rightarrow 0$ and $s_{2} \rightarrow 0$ are both realized, which means that the actual attack angle $\alpha$ will tend to the desired one $\alpha_{d}$.

Obviously, the derivation of Theorem 3 is carried out based on the condition of $\delta_{z}=\delta_{z d}$, which reflects that the servo-loop dynamics is not considered at this time. However, when the servo-loop dynamics is considered in the design, $\delta_{z}$ is not equal to $\delta_{z d}$ anymore; that is, $\delta_{z}=\delta_{z d}+d_{m}$. In fact, we can make $\left|d_{m}\right| \leq \Delta_{m}$ tenable by means of stable control system design, which will be presented in the next subsection.

3.2. With Consideration of Servo-Loop Dynamics. In this subsection, aiming at the three-layer design model (7), the dynamic surface attack angle control law can be designed as in the following steps.

Step B1. Design the robust surface variable for the 1st-layer subsystem:

$$
s_{1}=\alpha-\alpha_{d}
$$

Step B2. Calculate the virtual command of the 2nd-layer subsystem:

$$
\begin{array}{r}
\omega_{z 1 d}=-a_{1} \alpha+\dot{\alpha}_{d}-k_{1} s_{1}-q_{1} \operatorname{sgn}\left(s_{1}\right)-f_{\theta}, \\
k_{1}>0, \quad q_{1}>0 .
\end{array}
$$

Step B3. In order to get the differential information of $\omega_{z 1 d}$, we introduce a filter:

$$
\eta_{1} \dot{\omega}_{z 1 c}+\omega_{z 1 c}=\omega_{z 1 d}, \quad \omega_{z 1 c}(0)=\omega_{z 1 d}(0), \eta_{1}>0 .
$$

Step B4. Design the robust surface variable for the 2nd-layer subsystem:

$$
s_{2}=\omega_{z 1}-\omega_{z 1 c} .
$$

Step B5. Calculate the virtual command of the 3rd-layer subsystem:

$$
\begin{array}{r}
\delta_{z d}=\frac{-a_{2} \alpha-k_{2} s_{2}-q_{2} \operatorname{sgn}\left(s_{2}\right)+\dot{\omega}_{z 1 c}}{b_{2}} \\
k_{2}>0, \quad q_{2}>0 .
\end{array}
$$

Step B6. In order to get the differential information of $\delta_{z d}$, we introduce another filter:

$$
\eta_{2} \dot{\delta}_{z c}+\delta_{z c}=\delta_{z d}, \quad \delta_{z c}(0)=\delta_{z d}(0), \eta_{2}>0
$$

Step B7. Design the robust surface variable for the 3rd-layer subsystem:

$$
s_{3}=c\left(\delta_{z}-\delta_{z c}\right)+\dot{\delta}_{z}-\dot{\delta}_{z c}, \quad c>0 .
$$

Step B8. Calculate the command input of servo loop:

$$
\begin{array}{r}
\delta_{z d}{ }^{*}=\left(\left(2 \xi_{n} \omega_{n}-c\right) \dot{\delta}_{z}+\omega_{n}{ }^{2} \delta_{z}-k_{3} s_{3}\right. \\
\left.-q_{3} \operatorname{sgn}\left(s_{3}\right)+c \dot{\delta}_{z c}+\ddot{\delta}_{z c}\right)\left(\omega_{n}{ }^{2}\right)^{-1}, \\
k_{3}>0, \quad q_{3}>0 .
\end{array}
$$

Theorem 4. When the servo-loop dynamics is considered, by means of Steps B1-B8, the actual attack angle $\alpha$ will tend to the desired one $\alpha_{d}$, if the following conditions in (29) are all satisfied:

$$
\begin{aligned}
k_{1} k_{2} & >\frac{1}{4}, \\
q_{1} & >\Delta_{\alpha}+\Delta_{f \omega}, \\
q_{2} & >\Delta_{\omega}+\left|b_{2}\right| \Delta_{m}, \\
q_{3} & >\Delta_{\delta} .
\end{aligned}
$$

Proof. Choose a Lyapunov function as follows:

$$
V=\frac{1}{2} s_{1}^{2}+\frac{1}{2} s_{2}^{2}+\frac{1}{2} s_{3}^{2}
$$

Then, with the differential operation on (30), one can get

$$
\dot{V}=\dot{s}_{1} s_{1}+\dot{s}_{2} s_{2}+\dot{s}_{3} s_{3} \text {. }
$$

According to (7), (27), and (28), one can get

$$
\begin{aligned}
\dot{s}_{3}= & c\left(\dot{\delta}_{z}-\dot{\delta}_{z c}\right)+\ddot{\delta}_{z}-\ddot{\delta}_{z c} \\
= & c\left(\dot{\delta}_{z}-\dot{\delta}_{z c}\right)-2 \xi_{n} \omega_{n} \dot{\delta}_{z}-\omega_{n}^{2} \delta_{z}+\omega_{n}^{2} \delta_{z d}{ }^{*}+d_{\delta} \\
& -\ddot{\delta}_{z c}=-k_{3} s_{3}-q_{3} \operatorname{sgn}\left(s_{3}\right)+d_{\delta} .
\end{aligned}
$$

When the fourth condition in (29) is satisfied, we can get that $\dot{V}_{b}=\dot{s}_{3} s_{3} \leq 0$ always exists. Moreover, when and only when $s_{3}=0$ exists, $\dot{V}_{b}=0$ is tenable. Subsequently, we can further get $\delta_{z} \rightarrow \delta_{z c}$. Then, based on Assumption 2, we can conclude that there is $\delta_{z}=\delta_{z d}+d_{m}$ with $\left|d_{m}\right| \leq \Delta_{m}$.

Subsequently, as similar with the proof process of Theorem 3, we can also get the following equations:

$$
\begin{aligned}
& \dot{s}_{1}=\dot{\alpha}-\dot{\alpha}_{d}=s_{2}-k_{1} s_{1}-q_{1} \operatorname{sgn}\left(s_{1}\right)+d_{s 1} \\
& \dot{s}_{2}=\dot{\omega}_{z 1}-\dot{\omega}_{z 1 c}=-k_{2} s_{2}-q_{2} \operatorname{sgn}\left(s_{2}\right)+d_{s 2},
\end{aligned}
$$

where $d_{s 2}=d_{\omega}+b_{2} d_{m}$ and $\left|d_{s 2}\right| \leq \Delta_{s 2}=\Delta_{\omega}+\left|b_{2}\right| \Delta_{m}$.

According to (33), we can further get

$$
\begin{aligned}
\dot{V}_{a}= & \dot{s}_{1} s_{1}+\dot{s}_{2} s_{2} \\
= & {\left[s_{2}-k_{1} s_{1}-q_{1} \operatorname{sgn}\left(s_{1}\right)+d_{s 1}\right] s_{1} } \\
& +\left[-k_{2} s_{2}-q_{2} \operatorname{sgn}\left(s_{2}\right)+d_{s 2}\right] s_{2} \\
= & -k_{1} s_{1}{ }^{2}-k_{2} s_{2}{ }^{2}+s_{1} s_{2}-q_{1}\left|s_{1}\right|+d_{s 1} s_{1}-q_{2}\left|s_{2}\right| \\
& +d_{s 2} s_{2}
\end{aligned}
$$




$$
\begin{aligned}
\leq & -2 \sqrt{k_{1} k_{2}}\left|s_{1} s_{2}\right|+\left|s_{1} s_{2}\right|-q_{1}\left|s_{1}\right|+\Delta_{s 1}\left|s_{1}\right| \\
& -q_{2}\left|s_{2}\right|+\Delta_{s 2}\left|s_{2}\right| \\
= & -\left(2 \sqrt{k_{1} k_{2}}-1\right)\left|s_{1} s_{2}\right|-\left(q_{1}-\Delta_{s 1}\right)\left|s_{1}\right| \\
& -\left(q_{2}-\Delta_{s 2}\right)\left|s_{2}\right| .
\end{aligned}
$$

When the first three conditions in (29) are all satisfied, we can get that $\dot{V}_{a} \leq 0$ always exists. Moreover, when and only when both $s_{1}=0$ and $s_{2}=0$ coexist, $\dot{V}_{a}=0$ is tenable. As a result, $s_{1} \rightarrow 0$ and $s_{2} \rightarrow 0$ are also realized, which means that the actual attack angle $\alpha$ will tend to the desired one $\alpha_{d}$.

According to (8)-(12), we can calculate the servo-loop command $\delta_{z d}$ on the condition that the servo-loop dynamics is not considered. Meanwhile, according to (21)-(28), we can calculate new servo-loop command $\delta_{z d}{ }^{*}$ on the condition that the servo-loop dynamics is considered. This directly reflects the difference between the design processes without/with considering the servo-loop dynamics.

3.3. The Improved Control Laws. In order to avoid the highfrequency chattering at control input, which resulted from the signum term in control laws, the following saturation function will be employed to replace the signum function:

$$
\operatorname{sat}(s)=\left\{\begin{array}{ll}
\frac{s}{\kappa}, & |s| \leq \kappa, \\
\operatorname{sgn}(s), & |s|>\kappa,
\end{array} \quad \kappa>0 .\right.
$$

After introducing the saturation function (35), the final dynamic surface control laws without/with consideration of servo-loop dynamics can be, respectively and comprehensively, expressed by

$$
\begin{gathered}
s_{1}=\alpha-\alpha_{d}, \\
\omega_{z 1 d}=-a_{1} \alpha+\dot{\alpha}_{d}-k_{1} s_{1}-q_{1} \text { sat }\left(s_{1}\right)-f_{\theta}, \\
\eta \dot{\omega}_{z 1 c}+\omega_{z 1 c}=\omega_{z 1 d}, \quad \omega_{z 1 c}(0)=\omega_{z 1 d}(0), \\
s_{2}=\omega_{z 1}-\omega_{z 1 c}, \\
\delta_{z d}=\frac{-a_{2} \alpha-k_{2} s_{2}-q_{2} \operatorname{sat}\left(s_{2}\right)+\dot{\omega}_{z 1 c}}{b_{2}}, \\
s_{1}=\alpha-\alpha_{d}, \\
\omega_{z 1 d}=-a_{1} \alpha+\dot{\alpha}_{d}-k_{1} s_{1}-q_{1} \operatorname{sat}\left(s_{1}\right)-f_{\theta}, \\
\eta_{1} \dot{\omega}_{z 1 c}+\omega_{z 1 c}=\omega_{z 1 d}, \quad \omega_{z 1 c}(0)=\omega_{z 1 d}(0), \\
s_{2}=\omega_{z 1}-\omega_{z 1 c}, \\
\delta_{z d}=\frac{-a_{2} \alpha-k_{2} s_{2}-q_{2} \text { sat }\left(s_{2}\right)+\dot{\omega}_{z 1 c}}{b_{2}} \\
\eta_{2} \dot{\delta}_{z c}+\delta_{z c}=\delta_{z d}, \quad \delta_{z c}(0)=\delta_{z d}(0), \\
s_{3}=c\left(\delta_{z}-\delta_{z c}\right)+\dot{\delta}_{z}-\dot{\delta}_{z c}, \\
\delta_{z d}^{*}=\left(\left(2 \xi_{n} \omega_{n}-c\right) \dot{\delta}_{z}+\omega_{n}^{2} \delta_{z}-k_{3} s_{3}\right. \\
\left.-q_{3} \operatorname{sat}\left(s_{3}\right)+c \dot{\delta}_{z c}+\ddot{\delta}_{z c}\right)\left(\omega_{n}^{2}\right)^{-1}
\end{gathered} .
$$

In this section, the simulation results of the proposed control scheme are compared with those of conventional control scheme, which is designed without considering the servoloop dynamics.

In simulation, the parameters of researched aircraft are shown as follows: $V=2000 \mathrm{~m} / \mathrm{s}, m=1000 \mathrm{~kg}, \rho=$ $1.0 \mathrm{~kg} / \mathrm{m}^{3}, S=0.45 \mathrm{~m}^{2}, L=0.70 \mathrm{~m}$, and $J_{z 1}=5800 \mathrm{~kg} \cdot \mathrm{m}^{2}$. The nominal aerodynamic parameters are shown as follows: $C_{Y}^{\alpha}=57.26 \mathrm{rad}^{-1}, C_{Y}^{\delta_{z}}=3.63 \mathrm{rad}^{-1}, C_{z 1}^{\alpha}=-28.02 \mathrm{rad}^{-1}$, and $C_{z 1}^{\delta_{z}}=-37.97 \mathrm{rad}^{-1}$. Note that $10 \%$ perturbation of aerodynamic parameters will be considered in the actual plant. The parameters of servo loop are shown as follows: $\omega_{n}=100 \mathrm{rad} / \mathrm{s}$ and $\xi_{n}=0.3$. Moreover, taking the practical situation into consideration, the controller output is limited between \pm 35 deg.

In the selection process of control coefficients, the coefficients of low-pass filters and saturation functions can be first obtained. In concrete, the filter coefficients are selected based on the compromise between estimation accuracy on virtual command and suppression effect on "explosion of terms"; meanwhile the saturation function coefficients are selected based on the compromise between robust property of control system and smooth property of control output. As a result, we first select $\eta=\eta_{1}=\eta_{2}=0.001$ and $\kappa=0.1$. Then, the remainder coefficients in the two control schemes are all obtained by means of trial and errors method, and they are shown as follows.

When the servo-loop dynamics is not considered, the coefficients of attack angle control law are chosen as follows: $k_{1}=15, q_{1}=3, k_{2}=20$, and $q_{2}=2$.

When the servo-loop dynamics is considered, the coefficients of attack angle control law are chosen as follows: $k_{1}=$ $30, q_{1}=6, k_{2}=30, q_{2}=10, c=500, k_{3}=40$, and $q_{3}=5$.

The step response of servo loop is illustrated in Figure 1.

As seen from Figure 1, the nonideal dynamic characteristics of servo loop are obviously presented, including regulation time, overshoot, and stable errors, which will affect the working performance of the whole pitching control system.

With computer simulation on the whole pitching control system, the comparison curves of attack angles under the two control schemes are shown in Figure 2.

As seen from Figure 2, both the two robust control schemes can guarantee good tracking performances. However, when the servo-loop dynamics is considered in 


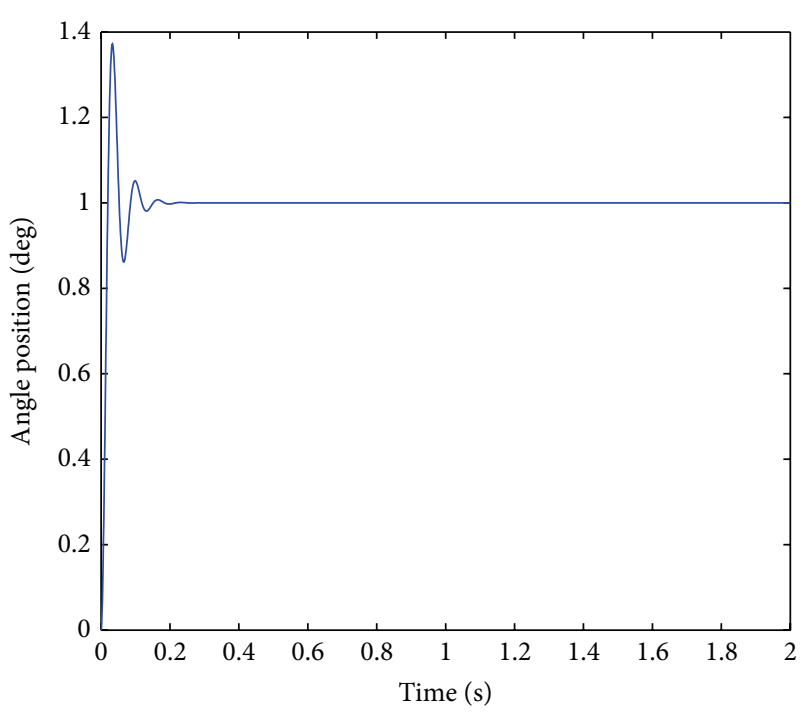

FIGURE 1: Step response curve of servo loop.

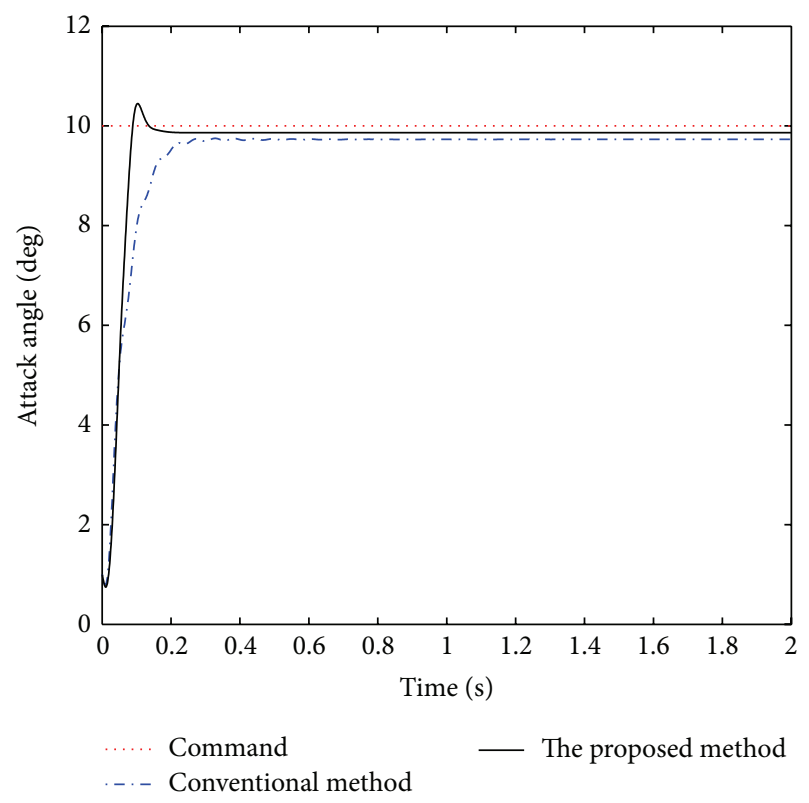

FIGURE 2: Curves of attack angles under the two control schemes.

the scheme design, the response speed of attack angle is faster, and the stable tacking errors are smaller.

Next, the outputs of servo loops under the two control schemes are shown in Figure 3.

As seen from Figure 3, the servo-loop output is smoother under the proposed control scheme, which is beneficial to the stability of the whole control system.

At this time, the control coefficients of the conventional scheme are chosen the same as with the proposed scheme; that is, $k_{1}=30, q_{1}=6, k_{2}=30$, and $q_{2}=10$, and then the tracking curve of attack angle under the conventional control scheme is shown in Figure 4.

As seen from Figure 4, when the control parameters of the conventional scheme are increased, the bad stability is

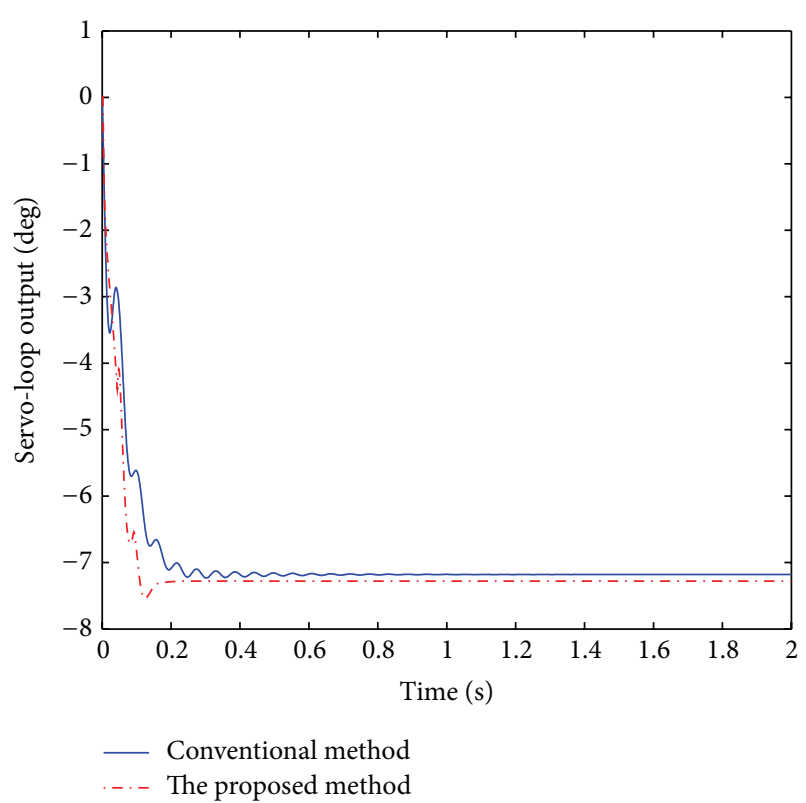

FIGURE 3: Output curves of servo loops under the two control schemes.

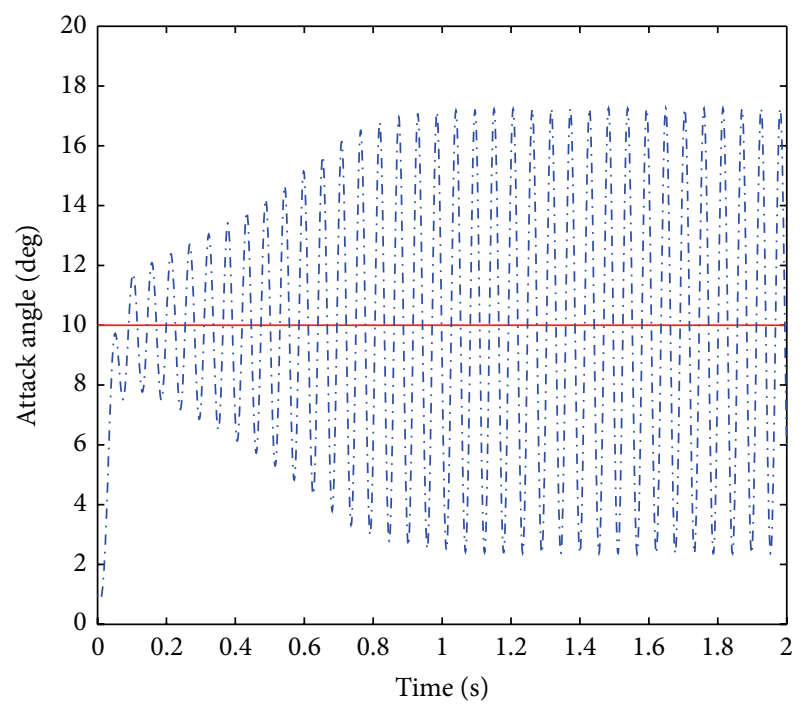

$\begin{array}{ll}\text {-. Command } & \text { Actual angle }\end{array}$

FIgURE 4: Tracking curve of attack angle under the conventional control scheme.

presented clearly, which reflects that the design margin can be effectively enhanced when the servo-loop dynamics is considered in the design process. Until now, the performance advantages under the proposed control scheme have been verified.

\section{Conclusions}

With consideration of second-order dynamics of electromechanical servo loop, this paper puts forward a robust attack 
angle control approach. By means of sliding mode control and dynamic surface control theories, the multiple subsystems of control plant are effectively connected, and the robust property of control system is also guaranteed. The stability analysis on attack angle control systems is given by using Lyapunov function method. From simulation results, we can see that the proposed scheme can guarantee that the attack angle control system possesses more satisfactory performances, compared with the conventional one which did not consider the servoloop dynamics in design period. Moreover, the structure of the proposed control scheme is simple; therefore it is easy to be realized in engineering application. In addition, the proposed method in this paper can be extended to explore the robust whole-channel attitude control approaches, which will be carried out in future works.

\section{Conflict of Interests}

The authors declare that there is no conflict of interests regarding the publication of this paper.

\section{Acknowledgment}

This work is supported by the National Nature Science Foundation of China (Grant no. 61403355).

\section{References}

[1] X. Liu, Y. Zhang, S. Wang, and W. Huang, "Backstepping attitude control for hypersonic gliding vehicle based on a robust dynamic inversion approach," Proceedings of the Institution of Mechanical Engineers, Part I: Journal of Systems and Control Engineering, vol. 228, no. 8, pp. 543-552, 2014.

[2] E. M. Wallner and K. H. Well, "Attitude control of a reentry vehicle with internal dynamics," in Proceedings of the AIAA Guidance, Navigation, and Control Conference and Exhibit, AIAA-2002-4647, Monterey, Calif, USA, August 2002.

[3] J. E. Stott and Y. B. Shtessel, "Launch vehicle attitude control using sliding mode control and observation techniques," Journal of the Franklin Institute: Engineering and Applied Mathematics, vol. 349, no. 2, pp. 397-412, 2012.

[4] M. Chen and B. Jiang, "Robust attitude control of near space vehicles with time-varying disturbances," International Journal of Control, Automation and Systems, vol. 11, no. 1, pp. 182-187, 2013.

[5] L. Sonneveldt, Q. P. Chu, and J. A. Mulder, "Nonlinear flight control design using constrained adaptive backstepping," Journal of Guidance, Control, and Dynamics, vol. 30, no. 2, pp. 322336, 2007.

[6] K. Z. Meguenni, M. Tahar, M. R. Benhadria, and Y. Bestaoui, "Fuzzy integral sliding mode based on backstepping control synthesis for an autonomous helicopter," Proceedings of the Institution of Mechanical Engineers, Part G: Journal of Aerospace Engineering, vol. 227, no. 5, pp. 751-765, 2013.

[7] D. Swaroop, J. K. Hedrick, P. P. Yip, and J. Gerdes, "Dynamic surface control for a class of nonlinear systems," IEEE Transactions on Automatic Control, vol. 45, no. 10, pp. 1893-1899, 2000.

[8] M. Hou and G. Duan, "Adaptive dynamic surface control for integrated missile guidance and autopilot," International Journal of Automation and Computing, vol. 8, no. 1, pp. 122-127, 2011.
[9] M. Hou, X. Liang, and G. Duan, "Adaptive block dynamic surface control for integrated missile guidance and autopilot," Chinese Journal of Aeronautics, vol. 26, no. 3, pp. 741-750, 2013.

[10] J. Zhang, Q. Li, N. Cheng, and B. Liang, "Adaptive dynamic surface control for unmanned aerial vehicles based on attractive manifolds," Journal of Guidance, Control, and Dynamics, vol. 36, no. 6, pp. 1776-1782, 2013.

[11] V. I. Utkin, "Variable structure systems with sliding modes," IEEE Transactions on Automatic Control, vol. 22, no. 2, pp. 212222,1977

[12] S. Laghrouche, F. Plestan, and A. Glumineau, "Higher-order sliding mode control based on integral sliding mode," Automatica, vol. 43, no. 3, pp. 531-537, 2007.

[13] X. Liu, Y. Wu, and B. Liu, "The research of adaptive sliding mode controller for motor servo system using fuzzy upper bound on disturbances," International Journal of Control, Automation and Systems, vol. 10, no. 5, pp. 1064-1069, 2012.

[14] W. Gao and J. C. Hung, "Variable structure control of nonlinear systems: a new approach," IEEE Transactions on Industrial Electronics, vol. 40, no. 1, pp. 45-55, 1993.

[15] M. I. Bayindir, H. Can, Z. H. Akpolat, M. Ozdemir, and E. Akin, "Application of reaching law approach to the position control of a vector controlled induction motor drive," Electrical Engineering, vol. 87, no. 4, pp. 207-215, 2005. 

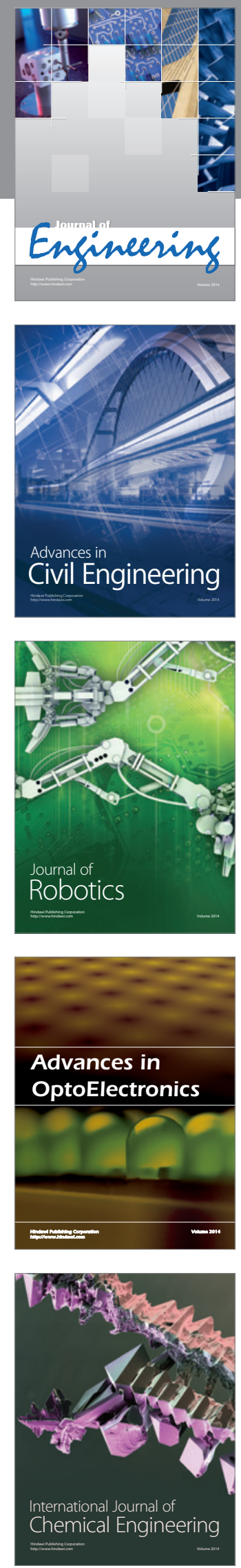

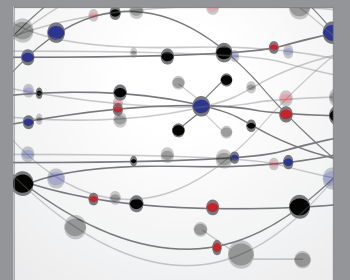

The Scientific World Journal
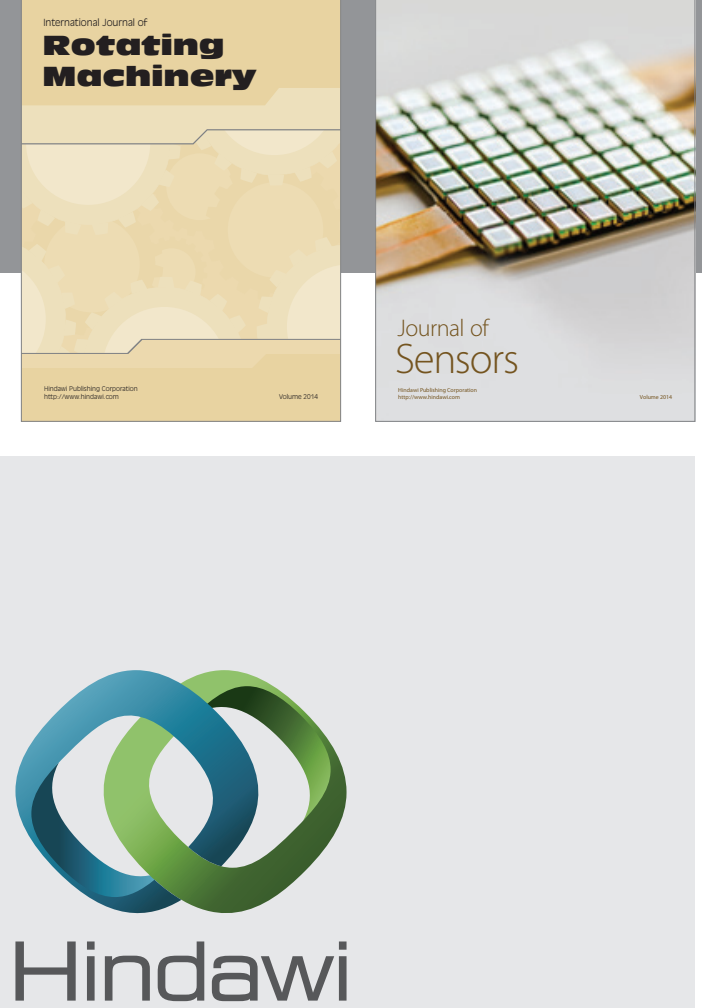

Submit your manuscripts at http://www.hindawi.com
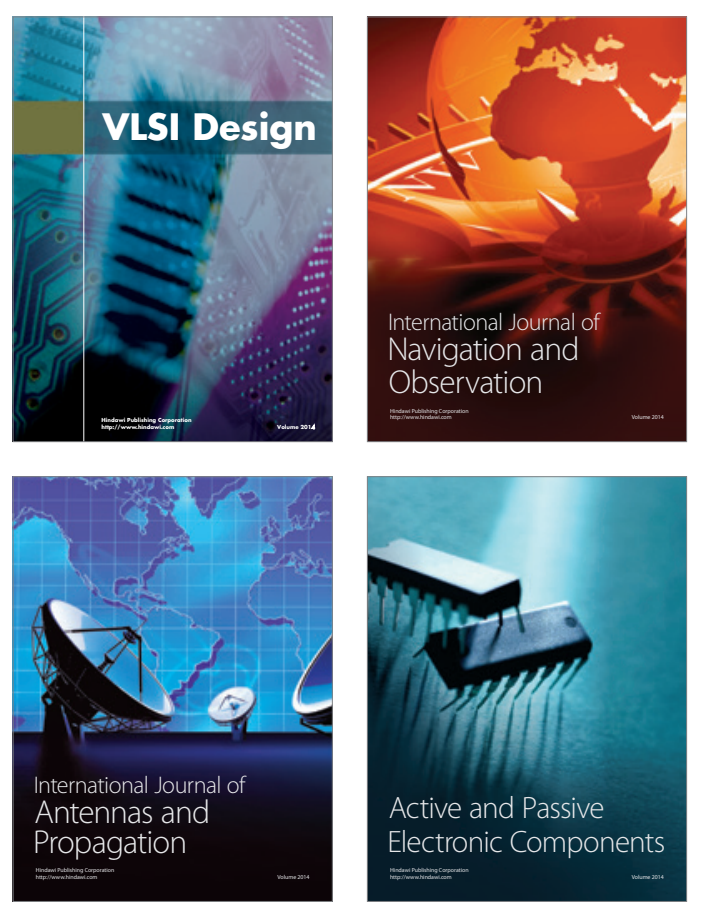
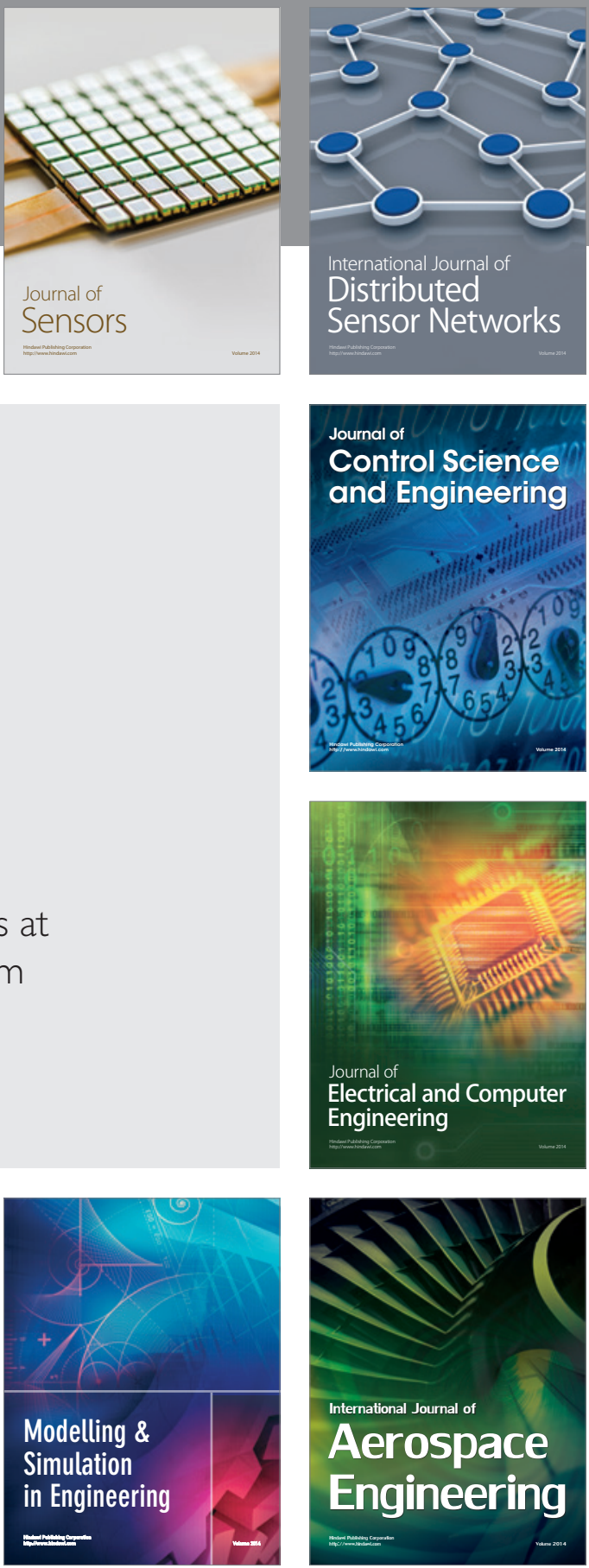

Journal of

Control Science

and Engineering
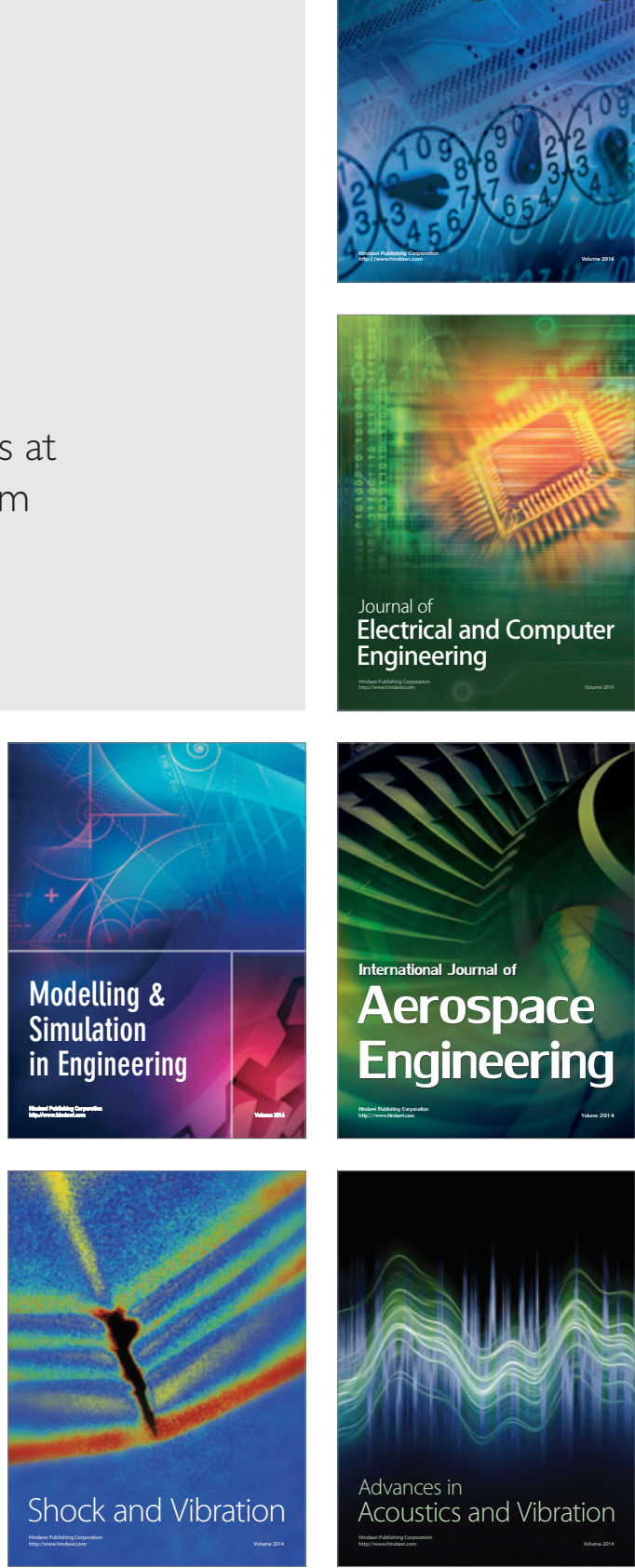